\title{
DIMENSIONS OF JET SCHEMES OF LOG SINGULARITIES
}

\author{
TAKEHIKO YASUDA
}

\begin{abstract}
We characterize Kawamata log terminal singularities and log canonical singularities by dimensions of jet schemes. Our main result is Theorem 2.4.
\end{abstract}

\section{INTRODUCTION}

For birational geometry, it is natural to consider a pair $(X, D)$ of a normal variety $X$ and a $\mathbb{Q}$-divisor on it, and to consider singularities of pairs. $K L T$ (Kawamata log terminal) and $L C$ (log canonical) singularities form important classes of log singularities. They are defined by using a log-resolution and discrepancies. When $X$ is $\mathbb{Q}$-Gorenstein, we can naturally define KLT and LC also for a pair $(X, q Y)$ where $Y$ is a closed subscheme of $X$ and $q \in \mathbb{Q}_{>0}$.

In Mus01, Mustaţă proposed a new point of view in the study of log singularities. He characterized KLT and LC pairs $(X, q Y)$ with $X$ smooth via dimensions of jet schemes of $Y$. The aim of this short paper is to extend his result to the case $X$ is $\mathbb{Q}$-Gorenstein and to shorten his proof. As he did, we also use the motivic integration as a main tool, which is invented by Kontsevich [Kon95] and extended to singular varieties by Denef and Loeser DL99a.

Acknowledgements. This paper forms a part of my master's thesis. First, I would like to thank Yujiro Kawamata for his encouragement and advise. I also thank Nobuyuki Kakimi for useful conversation.

\section{Motivic Integration}

In this section, we review the motivic integration invented by Kontsevich Kon95 and extended by Denef, Loeser DL99a. Craw's paper Cra99 is a nice introduction.

Let $k$ be an algebraically closed field of characteristic zero.

1.1. Extended Grothendieck rings of varieties. The Grothendieck ring of $k$-varieties, denoted $K_{0}(\operatorname{Var} / k)$, is the abelian group generated by the isomorphism classes $[X]$ of $k$-varieties with the relations $[X]=[X \backslash Y]+[Y]$ if $Y$ is a closed subvariety of $X$. The ring structure is defined by $[X][Y]=$ 
$[X \times Y]$. Let $\mathbb{L}$ be the class $\left[\mathbb{A}^{1}\right]$ of the affine line and let $\mathbb{M}$ be the localization $K_{0}(\operatorname{Var} / k)\left[\mathbb{L}^{-1}\right]$. We define the dimension of an element of $\mathbb{M}$ by

$$
\operatorname{dim}\left(\sum_{i}\left[X_{i}\right] \mathbb{L}^{m_{i}}\right):=\max _{i}\left(\operatorname{dim} X_{i}+m_{i}\right),
$$

with the convention $\operatorname{dim} \emptyset:=-\infty$. Then, the following hold: for $\alpha, \beta \in \mathbb{M}$,

- $\operatorname{dim} \alpha \beta=\operatorname{dim} \alpha+\operatorname{dim} \beta$,

- $\operatorname{dim}(\alpha+\beta) \leq \max \{\operatorname{dim} \alpha, \operatorname{dim} \beta\}$ and the equality holds if $\operatorname{dim} \alpha \neq$ $\operatorname{dim} \beta$.

If we set $F_{m} \mathbb{M}:=\{\alpha \in \mathbb{M} \mid \operatorname{dim} \alpha \leq m\}$, then $\left(F_{m} \mathbb{M}\right)_{m}$ is an ascending filtration with $F_{m} \mathbb{M} \cdot F_{n} \mathbb{M} \subset F_{m+n} \mathbb{M}$. We define the complete Grothendieck ring of $k$-varieties by

$$
\hat{\mathbb{M}}:=\lim _{\longleftarrow} \mathbb{M} / F_{m} \mathbb{M} \quad(m \rightarrow-\infty) .
$$

Any element $\alpha$ of $\hat{\mathbb{M}}$ is expressed as $\alpha=\sum_{m \in \mathbb{Z}} \alpha_{m}$ such that

- $\alpha_{m}=0$ or $\operatorname{dim} \alpha_{m}=m$,

- $\alpha_{m}=0$ for $m \gg 0$.

Then we define the dimension of $\alpha$ to be $\max \left\{m \mid \alpha_{m} \neq 0\right\}$.

We denote by $\hat{\mathbb{M}}^{\mathbb{Q}}$ the ring $\hat{\mathbb{M}}\left[\mathbb{L}^{q} ; q \in \mathbb{Q}\right]$. Any element of $\hat{\mathbb{M}}^{\mathbb{Q}}$ is expressed as $\alpha \mathbb{L}^{q}$ with $\alpha \in \hat{\mathbb{M}}, q \in \mathbb{Q}$. We define the dimension of $\alpha \mathbb{L}^{q}$ to be $\operatorname{dim} \alpha+q$.

1.2. Jet schemes and motivic measures. Let $X$ be a $k$-scheme. For $n \in \mathbb{Z}_{\geq 0} \cup\{\infty\}$, an $n$-jet on $X$ is a morphism

$$
\text { Spec } k[[t]] /\left(t^{n+1}\right) \rightarrow X,
$$

where we used the convention $\left(t^{\infty}\right)=(0) \subset k[[t]]$. The moduli scheme of the $n$-jets on $X$ always exists. We call it the $n$-jet scheme of $X$ and denote it by $L_{n}(X)$. If $X$ is of finite type, then for $n<\infty$, so is $L_{n}(X)$. If $X$ is smooth and of pure dimension $d$, then, for each $n \in \mathbb{Z}_{\geq 0}$, the natural projection $L_{n+1}(X) \rightarrow L_{n}(X)$ is a Zariski locally trivial $\mathbb{A}^{d}$-bundle.

Now assume that $X$ is a $k$-variety of pure dimension $d$. Let $\pi_{n}: L_{\infty}(X) \rightarrow$ $L_{n}(X)$ be the canonical projection.

Definition 1.1. A subset $A$ of $L_{\infty}(X)$ is stable at level $n$ if we have:

(1) $\pi_{n}(A)$ is a constructible subset of $L_{n}(X)$,

(2) $A=\pi_{n}^{-1} \pi_{n}(A)$,

(3) for any $m \geq n$, the projection $\pi_{m+1}(A) \rightarrow \pi_{m}(A)$ is a piecewise trivial $\mathbb{A}^{d}$-bundle.

(A morphism $f: Y \rightarrow X$ of schemes is called a piecewise trivial $\mathbb{A}^{d}$-bundle if there is a stratification $X=\coprod X_{i}$ such that $\left.f\right|_{f^{-1}\left(X_{i}\right)}: f^{-1}\left(X_{i}\right) \rightarrow X_{i}$ is isomorphic to $X_{i} \times \mathbb{A}^{d} \rightarrow X_{i}$ for each i.) A subset $A$ of $L_{\infty}(X)$ is stable if it is stable at level $n$ for some $n \in \mathbb{Z}_{\geq 0}$.

We see that the stable subsets of $L_{\infty}(X)$ constitute a Boolean algebra, that is, stable under finite intersection and finite union. For a stable subset 
$A$ of $L_{\infty}(X)$, an element $\left[\pi_{n}(A)\right] \mathbb{L}^{-n d} \in \hat{\mathbb{M}}$ is independent of the choice of $n \gg 0$. (We can define the class of a constructible set in $\mathbb{M}$ and $\hat{\mathbb{M}}$ in the evident fashion.) So the map

$$
\begin{aligned}
\mu_{X}:\left\{\text { stable subsets of } L_{\infty}(X)\right\} & \rightarrow \hat{\mathbb{M}} \\
A & \mapsto\left[\pi_{n}(A)\right] \mathbb{L}^{-n d}(n \gg 0)
\end{aligned}
$$

is a finite additive measure. We can extend $\mu_{X}$ to the family of the measurable subsets of $L_{\infty} X$, which is a family 'big enough'. For details, see [DL99b], Loo00]. We call $\mu_{X}$ the motivic measure on $L_{\infty} X$.

Let $A \subset L_{\infty}(X)$ be a measurable subset and $\nu: A \rightarrow \mathbb{Q} \cup\{\infty\}$ a function. We say that $\nu$ is a measurable function if the fibers are measurable and $\mu_{X}\left(\nu^{-1}(\infty)\right)=0$.

Definition 1.2. For a measurable function $\nu$, we formally define the motivic integration of $\mathbb{L}^{\nu}$ by

$$
\int_{A} \mathbb{L}^{\nu} d \mu_{X}:=\sum_{n \in \mathbb{Q}} \mu_{X}\left(\nu^{-1}(n)\right) \mathbb{L}^{n}
$$

We say that $\mathbb{L}^{\nu}$ is integrable if this infinite sum converges in $\hat{\mathbb{M}}^{\mathbb{Q}}$.

Let $Y \subset X$ be a closed subscheme and $\mathfrak{a}$ its ideal sheaf. A closed point $\gamma \in L_{\infty}(X)$ corresponds to a morphism $\gamma^{\prime}: \operatorname{Spec} k[[t]] \rightarrow X$. The function

$$
\begin{aligned}
F_{Y}: L_{\infty}(X) & \rightarrow \mathbb{Z}_{\geq 0} \cup\{\infty\} \\
\gamma & \mapsto n \text { if }\left(\gamma^{\prime}\right)^{-1} \mathfrak{a}=\left(t^{n}\right)
\end{aligned}
$$

is a measurable function. For a $\mathbb{Q}$-divisor $D=\sum_{i} q_{i} D_{i}$ on $X$ with $D_{i}$ a prime divisor, we define a measurable function $F_{D}:=\sum_{i} q_{i} F_{D_{i}}$. The following explicit formula give a way to compute motivic integrations.

Lemma 1.3. Assume that $X$ is smooth and $\sum_{i=1}^{s} D_{i}$ is a SNC divisor on $X$ with $D_{i}$ a prime divisor.

Let $m_{i} \in \mathbb{Z}_{\geq 0}, \quad(1 \leq i \leq s)$, and put $J:=\left\{i \mid m_{i}>0\right\} \subset\{1, \ldots, s\}$. Then we have

$$
\mu_{X}\left(\bigcap_{i} F_{D_{i}}^{-1}\left(m_{i}\right)\right)=\left[D_{J}^{\circ}\right](\mathbb{L}-1)^{|J|} \mathbb{L}^{-\sum m_{i}},
$$

where

$$
D_{J}^{\circ}:=\bigcap_{i \in J} D_{i} \backslash \bigcup_{i \in\{1, \ldots, s\} \backslash J} D_{i} .
$$

Proof. See Cra99, the proof of Thm. 2.15]. 


\section{MAIN THEOREM}

Let $X$ be a normal variety of dimension $d$. Assume that $X$ is $\mathbb{Q}$-Gorenstein, that is, for some $r \in \mathbb{Z}_{>0}, r K_{X}$ is a Cartier divisor. For a resolution $p: \tilde{X} \rightarrow X$, the relative canonical divisor $K_{\tilde{X} / X}$ is

$$
K_{\tilde{X} / X}:=\frac{1}{r}\left(r K_{\tilde{X}}-p^{*}\left(r K_{X}\right)\right)
$$

Let $Y$ be a closed subscheme of $X$ and $\mathfrak{a}$ its ideal sheaf. By Hironaka's theorem, there are a resolution $p: \tilde{X} \rightarrow X$ and a SNC divisor $\sum_{i} D_{i}$ on $\tilde{X}$ such that

- $p^{-1} \mathfrak{a}=\mathcal{O}_{\tilde{X}}\left(-\sum_{i} y_{i} D_{i}\right)$ for some $y_{i} \in \mathbb{Z}_{\geq 0}$,

- $K:=K_{\tilde{X} / X}=\sum_{i} a_{i} D_{i}$ for some $a_{i} \in \mathbb{Q}$.

Fix these notations through the rest of the paper. In the proof of Theorem 2.4, we suppose additional conditions on $p$.

Definition 2.1. For $q \in \mathbb{Q}_{>0}$, we say that the pair $(X, q Y)$ is $K L T$ (Kawamata $\log$ terminal), resp. $L C$ (log canonical) if for every $i,-q y_{i}+a_{i}+1>0$, resp. $-q y_{i}+a_{i}+1 \geq 0$.

Let $X_{\text {reg }}$ be the smooth locus of $X$ and $\iota: X_{\text {reg }} \hookrightarrow X$ the inclusion. Then $\omega_{X}^{[r]}=\iota_{*}\left(\left(\Omega_{X}^{d}\right)^{\otimes r}\right)$ is an invertible sheaf. We define an ideal sheaf $\mathcal{J} \subset \mathcal{O}_{X}$ by the following equation:

$$
\mathcal{\partial} \omega_{X}^{[r]}=\operatorname{Image}\left(\left(\Omega_{X}^{d}\right)^{\otimes r} \rightarrow \omega_{X}^{[r]}\right) .
$$

Let $Z \subset X$ be the closed subscheme associated to $\mathcal{J}$. Then $\operatorname{Supp} Z=X_{\text {sing }}$. The following is a variation of the transformation rule, a key of the proof of the main theorem.

Theorem 2.2. Let $A \subset L_{\infty} X$ be a measurable subset and $\nu: A \rightarrow \mathbb{Q} \cup\{\infty\}$ a measurable function. Then we have the following equality:

$$
\int_{A} \mathbb{L}^{\nu+(1 / r) \cdot F_{Z}} d \mu_{X}=\int_{p_{\infty}^{-1}(A)} \mathbb{L}^{\nu \circ p_{\infty}-F_{K}} d \mu_{\tilde{X}}
$$

Proof. It is a direct consequence of the transformation rule DL99a, Lem. 3.3].

For each $e \in \mathbb{Z}_{\geq 0}$, we put $A_{e}:=F_{Z}^{-1}(e)$. For each $n, e \in \mathbb{Z}_{\geq 0}$, we define

$$
L_{n}^{e}(Y):=L_{n}(Y) \cap \pi_{n}\left(A_{e}\right),
$$

where we take the intersection in $L_{n}(X)$. Of course, $L_{n}^{e}(Y)$ depends on the inclusion $Y \subset X$.

Lemma 2.3. There is a positive integer $\theta$ such that for any $n, e \in \mathbb{Z}_{\geq 0}$ with $n \geq \theta e$, the natural projection $\pi_{n+1}\left(A_{e}\right) \rightarrow \pi_{n}\left(A_{e}\right)$ is a piecewise trivial $\mathbb{A}^{d}$-bundle.

Proof. See [DL99a, Lem. 4.1]. 
Theorem 2.4. Suppose $\operatorname{Supp} Z \supset X_{\text {sing }}$. Let $\theta$ be a positive integer as in Lemma 2.3. For $l \in \mathbb{Z}_{>0}$, let $l Y$ be the closed subscheme of $X$ associated to the ideal sheaf $\mathfrak{a}^{l}$. Suppose that $\mathfrak{a}^{l} \subset \mathrm{J}^{\theta}$. Then,

(1) $(X, q Y)$ is KLT iff for any $e, n \in \mathbb{Z}_{\geq 0}$ with $n \geq \theta e$,

$$
\operatorname{dim} L_{n}^{e}(l Y)+e / r<(n+1)(d-q / l) .
$$

(2) $(X, q Y)$ is LC iff for any $e, n \in \mathbb{Z}_{\geq 0}$ with $n \geq \theta e$,

$$
\operatorname{dim} L_{n}^{e}(l Y)+e / r \leq(n+1)(d-q / l) .
$$

Proof. We prove only (1). For each $n \in \mathbb{Z}_{\geq 0}$, let $B_{n}:=F_{Y}^{-1}(n)$ and $B_{\geq n}:=$ $F_{Y}^{-1}\left(\mathbb{Z}_{\geq n}\right)$. For each $e, n \in \mathbb{Z}_{\geq 0}$ with $n \geq \theta e$, consider the following element of $\hat{M}^{\mathbb{Q}}$ :

$$
\begin{aligned}
S(e, n) & :=\int_{B_{n+1} \cap A_{e}} \mathbb{L}^{q F_{Y}+(1 / r) \cdot F_{Z}} d \mu_{X} \\
& =\mu_{X}\left(B_{n+1} \cap A_{e}\right) \mathbb{L}^{(n+1) q+e / r}
\end{aligned}
$$

By Lemma 2.3.

$$
\begin{aligned}
S(e, n) & \left.=\left(\mu_{X}\left(B_{\geq n+1} \cap A_{e}\right)-\mu_{X}\left(B_{\geq n+2}\right) \cap A_{e}\right)\right) \mathbb{L}^{(n+1) q+e / r} \\
& =\left(\left[L_{n}^{e}(Y)\right]-\left[L_{n+1}^{e}(Y)\right] \mathbb{L}^{-d}\right) \mathbb{L}^{-n d+(n+1) q+e / r} .
\end{aligned}
$$

By Lemma 2.3 again, we have $\operatorname{dim} L_{n+1}^{e}(Y) \leq \operatorname{dim} L_{n}^{e}(Y)+d$, and hence

$$
\operatorname{dim} S(e, n) \leq \operatorname{dim} L_{n}^{e}(Y)-n d+(n+1) q+e / r .
$$

If the equality does not hold, then $\operatorname{dim} L_{n+1}^{e}(Y)=\operatorname{dim} L_{n}^{e}(Y)+d$.

We suppose that $p^{-1} \mathcal{J}=\mathcal{O}_{\tilde{X}}\left(-\sum_{i} z_{i} D_{i}\right)$ with $z_{i}>0$.

We set $\tilde{Y}:=\sum_{i} y_{i} D_{i}$ and $\tilde{Z}:=\sum_{i} z_{i} D_{i}$. From Theorem 2.2, we have

$$
S(e, n)=\int \mathbb{L}^{q F_{\tilde{Y}}-F_{K}} d \mu_{\tilde{X}}
$$

where the domain of the integration is

$$
F_{\tilde{Y}}^{-1}(n+1) \cap F_{\tilde{Z}}^{-1}(e) .
$$

From Lemma 1.3, we obtain

$$
S(e, n)=\sum_{J \subset\{1, \ldots, s\}} \sum_{\mathbf{m} \in M}\left[D_{J}^{\circ}\right](\mathbb{L}-1)^{|J|} \mathbb{L}^{-\sum\left(-q y_{i}+a_{i}+1\right) m_{i}},
$$

where

$$
\begin{aligned}
M & =M(J, n, e) \\
& :=\left\{\mathbf{m}=\left(m_{i}\right)_{i \in J} \in\left(\mathbb{Z}_{>0}\right)^{J} \mid \sum y_{i} m_{i}=n \text { and } \sum z_{i} m_{i}=e\right\} .
\end{aligned}
$$

For each $J$ and $\mathbf{m}$, the highest term of

$$
\left[D_{J}^{\circ}\right](\mathbb{L}-1)^{|J|} \mathbb{L}^{-\sum\left(-q y_{i}+a_{i}+1\right) m_{i}}
$$


has a positive coefficient. Hence,

$$
\operatorname{dim} S(e, n)=\max _{\substack{J \subset\{1, \ldots, s\} \\ \mathbf{m} \in M}}\left(d-\sum_{i}\left(-q y_{i}+a_{i}+1\right) m_{i}\right) .
$$

'Only if' part: The proof is by contradiction. So assume that $(X, q Y)$ is KLT, that is, for every $i,-q y_{i}+a_{i}+1>0$, and that for some $n^{\prime}, e^{\prime} \in \mathbb{Z}_{\geq 0}$ with $n^{\prime}>\theta e^{\prime}$,

$$
\operatorname{dim} L_{n^{\prime}}^{e^{\prime}}(Y)+e^{\prime} / r \geq\left(n^{\prime}+1\right)(d-q) .
$$

By (2.2), we have $\operatorname{dim} S\left(e^{\prime}, n^{\prime}\right)<d$. On the other hand, since

$$
\operatorname{dim} L_{n^{\prime}}^{e^{\prime}}(Y)-n^{\prime} d+\left(n^{\prime}+1\right) q+e^{\prime} / r \geq d,
$$

the equality in (2.1) does not hold. Hence $\operatorname{dim} L_{n^{\prime}+1}^{e^{\prime}}(Y)=\operatorname{dim} L_{n^{\prime}}^{e^{\prime}}(Y)+d$ and hence $\operatorname{dim} L_{n^{\prime}+1}^{e^{\prime}}(Y)+e^{\prime} / r \leq\left(\left(n^{\prime}+1\right)+1\right)(d-q)$. By the same argument, we have $\operatorname{dim} L_{n^{\prime}+2}^{e^{\prime}}(Y)=\operatorname{dim} L_{n^{\prime}+1}^{e^{\prime}}(Y)+d$ and so on. It contradicts Lemma 2.5 .

'If' part: From inequality (2.1), we have that for any $e, n$ with $n \geq \theta e$,

$$
\operatorname{dim} S(e, n)<d \text {. }
$$

In view of (2.2) and (2.3), it is easy to find that for any $i,-q y_{i}+a_{i}+1>0$, that is, $(X, q Y)$ is KLT. We have thus completed the proof.

Lemma 2.5. Then for each $e \in \mathbb{Z}_{\geq 0}$, there is a real numbers $b$ such that $b<d$ and for every $n \in \mathbb{Z}_{\geq 0}$,

$$
\operatorname{dim} L_{n}^{e}(Y) \leq b n+(\text { const }) .
$$

Proof. It suffices to find an increasing linear function $\psi: \mathbb{Z}_{\geq 0} \rightarrow \mathbb{Z}_{\geq 0}$ and a real number $b<d$ such that

$$
\operatorname{dim} L_{\psi(n)}^{e}(Y) \leq b \psi(n)+\text { (const) } .
$$

First, the case $Y$ reduced: For $m, n \in \mathbb{Z}_{\geq 0}$ with $m \geq n$, we denote by $\pi_{n}^{m}$ the natural projection $L_{m}(X) \rightarrow L_{n}(X)$. By Greenberg's theorem Gre66, Cor. 1], there is a linear function $g: \mathbb{Z}_{\geq 0} \rightarrow \mathbb{Z}_{\geq 0}$ such that

- for every $n \in \mathbb{Z}_{\geq 0}, g(n) \geq n$,

$$
\text { - } \pi_{n}\left(L_{\infty}(Y)\right)=\pi_{n}^{g(n)}\left(L_{g(n)}(Y)\right) \text {. }
$$

Since $L_{g(n)}^{e}(Y) \subset\left(\pi_{n}^{g(n)}\right)^{-1} \pi_{n}^{g(n)}\left(L_{g(n)}^{e}(Y)\right)$,

$$
\operatorname{dim} L_{g(n)}^{e}(Y) \leq \operatorname{dim}\left(\pi_{n}^{g(n)}\right)^{-1} \pi_{n}^{g(n)}\left(L_{g(n)}^{e}(Y)\right) .
$$

By the definition of $g$,

$$
\pi_{n}^{g(n)}\left(L_{g(n)}^{e}(Y)\right)=\pi_{n}\left(L_{\infty}^{e}(Y)\right)
$$

Let $d^{\prime}$ be the dimension of $Y$. By [DL99a, Lem 4.3],

$$
\operatorname{dim} \pi_{n}^{g(n)}\left(L_{g(n)}^{e}(Y)\right)=\operatorname{dim} \pi_{n}\left(L_{\infty}^{e}(Y)\right) \leq \operatorname{dim}(n+1) d^{\prime} .
$$


Hence, by Lemma 2.3,

$$
\begin{aligned}
\operatorname{dim} L_{g(n)}^{e}(Y) & \leq(n+1) d^{\prime}+(g(n)-n) d+(\text { const }) \\
& =g(n) d+n\left(d^{\prime}-d\right)+(\text { const }) \\
& =g(n)\left(d+\left(d^{\prime}-d\right) / C_{1}\right)+(\text { const }),
\end{aligned}
$$

where $C_{1}$ is the constant such that $g(n)=C_{1} n+$ (const). We have thus proved the assertion in this case.

The general case: It suffices to show the case where $Y=l\left(Y_{\text {red }}\right)$ for some $l \in \mathbb{Z}_{>0}$. By the definitions, we have the following

$$
\begin{aligned}
L_{l n-1}^{e}(Y) & =\pi_{l n-1}\left(F_{Y}^{-1}(l n) \cap A_{e}\right), \\
L_{n-1}^{e}\left(Y_{\text {red }}\right) & =\pi_{n-1}\left(F_{Y_{\text {red }}}^{-1}(n) \cap A_{e}\right) .
\end{aligned}
$$

Because for $n$, $e$ with $n \geq \theta e, F_{Y}^{-1}(\ln ) \cap A_{e}=F_{Y_{\text {red }}}^{-1}(n) \cap A_{e}$ is stable at level $n$, we have $L_{l n-1}^{e}(Y)=\left(\pi_{n-1}^{l n-1}\right)^{-1} L_{n}^{e}\left(Y_{\text {red }}\right)$. Therefore, for some $b<d$,

$$
\begin{aligned}
\operatorname{codim}\left(L_{l n-1}^{e}(Y) / \pi_{l n-1}\left(A_{e}\right)\right) & =\operatorname{codim}\left(L_{n-1}^{e}\left(Y_{\text {red }}\right) / \pi_{n-1}\left(A_{e}\right)\right) \\
& \geq(d-b) \varphi(n)+(\text { const }) .
\end{aligned}
$$

Because $\operatorname{dim} \pi_{n}\left(A_{e}\right)=d n+$ (const), we have

$$
\begin{aligned}
\operatorname{dim} L_{l n-1}^{e}(Y) & \leq d l n-(d-b) n+(\text { const }) \\
& \leq(d-(d-b) / l)(l n-1)+(\text { const }) .
\end{aligned}
$$

This completes the proof.

\section{REFERENCES}

[Cra99] Alastair Craw. An introduction to motivic integration. preprint, math. AG/9911179, 1999.

[DL99a] Jan Denef and François Loeser. Germs of arcs on singular algebraic varieties and motivic integration. Invent. Math., 135(1):201-232, 1999.

[DL99b] Jan Denef and François Loeser. Motivic integration, quotient singularities and the McKay correspondence. preprint, math.AG/9903187, 1999.

[Gre66] Marvin J. Greenberg. Rational points in Henselian discrete valuation rings. Publ. Math. I.H.E.S., 31:59-64, 1966.

[Kon95] Maxim Kontsevich. Lecture at orsay. 1995.

[Loo00] Eduard Looijenga. Motivic measures. preprint, math. AG/0006220, 2000.

[Mus01] Mircea Mustaţă. Singularities of Pairs via Jet Schemes. preprint, math.AG/0102201, 2001.

Department of Mathematical Sciences, University of Tokyo, Komaba, Meguro, TOKYO, 153-8914, JAPAN

E-mail address: t-yasuda@ms.u-tokyo.ac.jp 